\title{
ANALYSIS OF LONG-TERM AAVSO OBSERVATIONS OF RS OPHIUCHI
}

\author{
B.D. OPPENHEIMER AND J.A. MATTEI \\ American Association of Variable Star Observers \\ 25 Birch Street \\ Cambridge, Massachusetts 02138 \\ U.S.A.
}

\begin{abstract}
We present here a study of the five reported outbursts of the recurrent nova RS Ophiuchi, together with an analysis of its light variation between outbursts. We find that the shapes and decline rates of the outbursts are strikingly similar to each other. We note the possibility of an additional, unreported outburst in 1945, supported by the similarity of its decline to that of other outbursts. Each interval between outbursts shows periodicity within itself, however, there is no periodicity common to all, and the possible periods range between 892 and 2283 days.
\end{abstract}

\section{Introduction}

RS Ophiuchi is a cataclysmic variable of the recurrent nova type. It is an interacting binary system consisting of an $\mathrm{M}$ red giant and a blue compact companion (Barbon, Mammano \& Rosino 1968; Sanduleak \& Stephenson 1973). Due to its composite spectrum it has also been classified as a symbiotic star (Barbon et al. 1968). Its orbital period has been derived from radial-velocity variations (Dobrzycka \& Kenyon 1994) to be 460 days, and its distance has been estimated to be $1.6 \mathrm{kpc}$ (Hjellming et al. 1986).

It has had five reported outbursts, in 1898, 1933, 1958, 1967, and 1985. All of the outbursts show very similar light curves.

RS Ophiuchi is the only known recurrent nova that shows significant light fluctuations between outbursts. In this study we analyzed the longterm behavior of RS Ophiuchi both during outbursts and between outbursts, using mostly AAVSO observations spanning 104 years. 


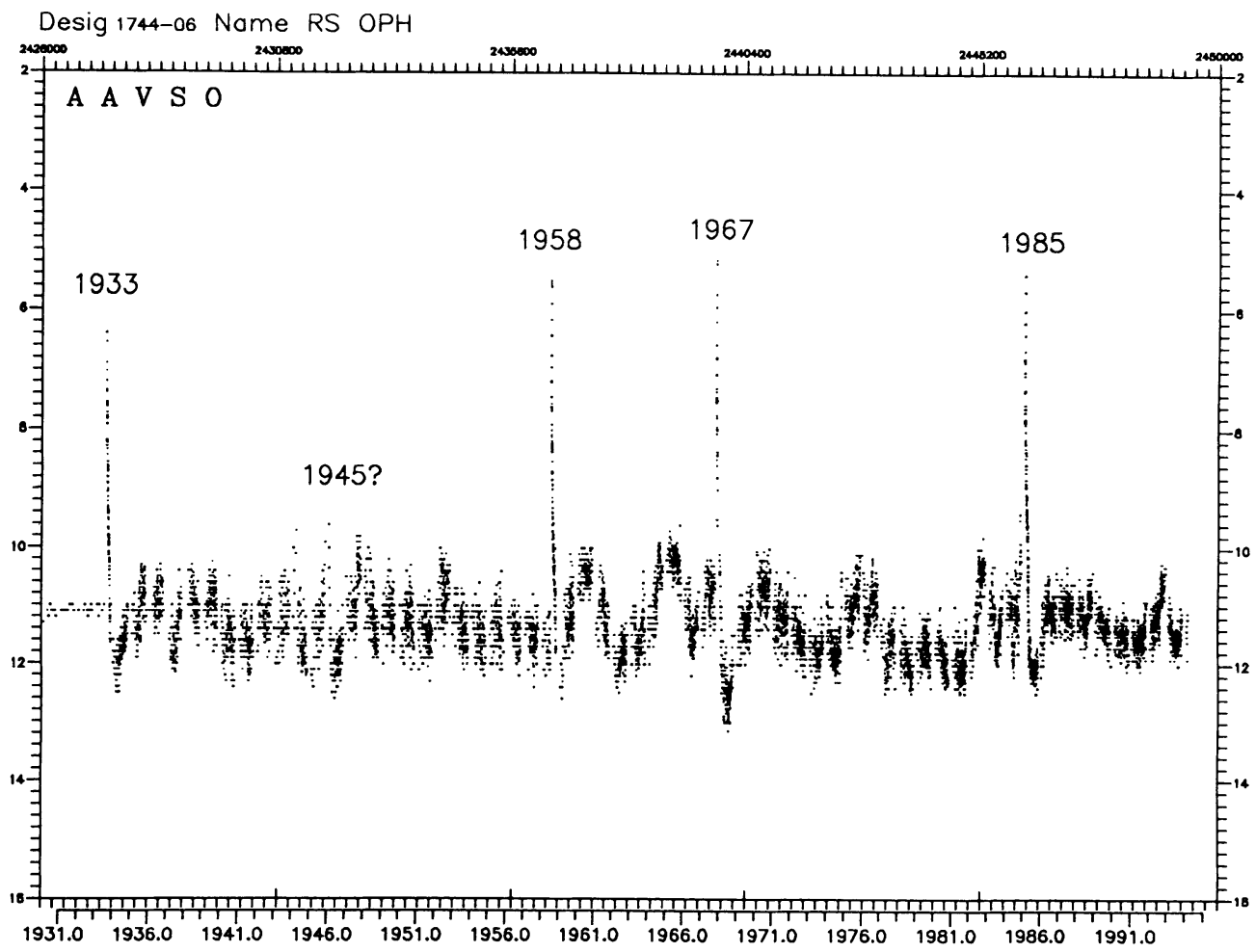

Figure 1. Light curve of AAVSO observations of RS Ophiuchi from 1930 to mid-1993, plotted date (top - Julian Day, bottom - year) vs. visual magnitude. 


\section{Observations and Analysis}

RS Ophiuchi has been in the observing program of AAVSO and well-monitored by hundreds of observers since its 1933 outburst (Fig. 1). For this study we have utilized 26966 visual observations from the AAVSO International Database and Archives between 1920 and May 1993, and 565 photographic observations from Harvard plates from 1890 to 1916 (Campbell 1920). We have studied the outbursts and the intervals between them separately. We used 0.5-day means of the observations for the outbursts and 1-day means for the non-outburst intervals.

\subsection{OUTBURSTS}

An outburst is defined as the sudden rise in brightness from quiescent state to about 5th magnitude within 24 hours. Each outburst was studied individually, and then superimposed onto each other. Three stages of decline were found from maximum to minimum. These stages were isolated and decline rates obtained for each of them. Particular attention was given to the interval following an observing gap, beginning in December 1945, when the star was observed to be bright at magnitude 9.6. This interval was studied thoroughly and the decline rate of the light curve was compared with the rest of the outbursts to check whether this may have been an outburst caught at the tail-end.

\subsection{INTERVALS BETWEEN OUTBURSTS}

Six intervals between outbursts from 1898 to 1993 were identified and each interval was analyzed using the Date-Compensated Discrete Fourier (DCDF) Analysis technique (Ferraz-Mello 1981) developed for MS-DOS at AAVSO. For each interval, a primary Fourier transform was taken, followed by a residual and a second residual, to find the possible strong periods. Particular attention was given in search of both the 230-day period and the 460-day spectroscopic orbital periods reported by Garcia (1986), then later revised by Dobrzycka \& Kenyon (1994).

\section{Discussion}

\subsection{OUTBURSTS}

Each of the five reported outbursts is strikingly similar to each other. In each outburst, the rise to maximum is fast, as the star brightens by about 6 magnitudes within 24 hours, reaching maximum visual magnitude of about 5.0 ; the star then immediately starts to fade. The decline from an outburst is in three stages. The first stage is the fastest, averaging $0.09 \mathrm{mag} \mathrm{day}^{-1}$ 
and lasting 43 days, followed by a decline rate of only $0.02 \mathrm{mag} \mathrm{day}^{-1}$ until 85 days after outburst. The average rate of decline of the final stage is 0.05 mag day $^{-1}$ lasting until 122 days after outburst (see Table 1).

TABLE 1. Outbursts of RS Ophiuchi

\begin{tabular}{lllllllll}
\hline $\begin{array}{l}\text { Outburst } \\
\text { Year }\end{array}$ & $\begin{array}{l}\text { JD } \\
\text { Max } \\
(2400000+)\end{array}$ & $\begin{array}{l}\text { Max } \\
\mathrm{mag}\end{array}$ & $\Delta t(1)$ & $\begin{array}{l}\text { Rate of } \\
\Delta t(1) \\
\left(\mathrm{mag} \mathrm{d}^{-1}\right)\end{array}$ & $\begin{array}{l}\Delta t(2) \\
(\text { days })\end{array}$ & $\begin{array}{l}\text { Rate of } \\
\Delta t(2) \\
\left(\mathrm{mag} \mathrm{d}^{-1}\right)\end{array}$ & $\begin{array}{l}\Delta t(3) \\
(\text { days })\end{array}$ & $\begin{array}{l}\text { Rate of } \\
\Delta t(3) \\
\left(\mathrm{mag} \mathrm{d}^{-1}\right)\end{array}$ \\
\hline 1898 & $14442 ?$ & - & 51 & $.06 ?$ & 112 & $.01 ?$ & 128 & $.06 ?$ \\
1933 & 27297 & $6.4 ?$ & 48 & $.06 ?$ & 81 & .02 & 118 & $.06 ?$ \\
$1945 ?$ & $31786 ?$ & -- & - & - & 76 & - & 118 & $.05 ?$ \\
1958 & 36399 & 5.4 & 41 & .10 & 89 & .02 & 117 & .04 \\
1967 & 39791 & 4.9 & 39 & $.12 ?$ & 89 & $.02 ?$ & 140 & .04 \\
1985 & 46094 & 5.4 & 38 & .10 & 65 & .02 & 111 & .05 \\
\hline Average & & 5.5 & 43 & .09 & 85 & .02 & 122 & .05 \\
\hline
\end{tabular}

During the secondary decline phase, the star brightens slightly and the decline slows down. This interval coincides with the appearance of the coronal lines of [Fe X], [Fe XIV], and [A X], forbidden lines of [O III] and [N II], and permitted lines of N III, HeI, and HeII in the spectrum of each outburst (Barbon, Mammano \& Rosino 1968; Hack \& Selvelli 1993). The appearance of some of the coronal lines in the visual spectra, such as [Fe X] and [Fe XIV], may cause the overall visual brightness to increase. As they weaken the star fades and continues to decline at the third, faster rate.

The star reaches minimum at about magnitude 12.5 , on the average 216 days after outburst. It then brightens, reaching a mean maximum of magnitude 10.3 in 501 days, or 717 days after outburst maximum.

\subsubsection{Outburst in 1945}

There is strong evidence in the AAVSO observations that RS Ophiuchi had another outburst in December 1945 (JD 2431786) which has not been reported previously. An observation made at magnitude 9.9 on JD 2431784, 2 days before the possible maximum outburst, may have been the beginning of the rise to maximum. For the next 68 days, the star was in its seasonal observing gap. On JD 2431854, 65 days after the possible maximum, it was observed at magnitude 9.6. Over the next 69 days, the brightness fell 2.9 magnitudes, a rate of change never before observed in this star for such an extended amount of time outside outbursts. The light curve and the rate of decline is similar to the third stage of other outbursts. After reaching 
minimum the star brightened by 2.5 magnitudes in 521 days, a behavior also seen in other outbursts. Thus, we strongly believe that RS Ophiuchi indeed had another outburst in 1945 which has not been reported in the literature until now. The occurrence of this outburst may have bearing on the modeling of the system in that with the 5 known outbursts, the average interval between outbursts is 22 years, whereas with an outburst in 1945, the average reduces to 13 years between the 1933 and 1985 outbursts. With so few observations between 1898 and 1933 , one or more outbursts could have been missed, thus this interval has not been included in the averages.

\subsection{INTERVALS BETWEEN OUTBURSTS}

The light curve outside of the outbursts resembles the light curves of other symbiotic stars such as Z Andromedae and CH Cygni. These stars show significant oscillations with multiple periods.

The intervals between outbursts of RS Ophiuchi vary from each other both in the amplitude of variation, ranging between one and three magnitudes, and in the complexity of the modulations. The AAVSO light curve from 1930 to May 1993 (Fig. 1) shows the longterm behavior and the activity between outbursts clearly. The intervals between the 1958-1967 and the 1967-1985 outbursts appear to be more active, showing the largest range of variation.

After the decline of each outburst, there is a secondary brightening, reaching a minor-maximum in the average 717 days after the outburst. This is usually followed by two or three similar modulations until the next outburst.

The Date-Compensated Discrete Fourier Transform (DCDFT) applied to each of the intervals indicates that there is no common period between these intervals. Modulations between outbursts are multiperiodic, with the possible periods ranging from 892 to 2283 days. The whole data set, excluding the outbursts, was also studied, and the DCDFT applied to this set indicates a period of 2016 days.

A likely period of 471 days was found for the time interval 1985 to 1993 . This period is within the errors of the 460-day orbital period derived by Dobrzycka \& Kenyon (1994). No period at or close to 260 days (Garcia 1986) was found by the DCDFT.

Further analysis of the AAVSO light curves is being carried out in search of a 50 to 70-day period reported by Tempesti (1975) in the photoelectric data between 1972 and 1973. 


\section{Conclusions}

The outburst light curves are very similar to each other and show three stages of decline. The first stage is the fastest, the second is slower, and the third is intermediate in rate of decline. There is minor brightening during the second stage that may coincide with the appearance of coronal lines in the spectra.

The outbursts may be phase-dependent, occurring near the minimum of a modulation and followed by rise to secondary minor-maximum after each outburst. The mechanism causing the modulations, particularly the longer ones, may be connected to the occurrence of the outbursts.

There is strong evidence in the AAVSO data of an unreported outburst in 1945.

RS Ophiuchi shows significant fluctuation between outbursts, varying between magnitude 9.6 and and 12.8. The light variation shows a wide variety of behavior from one interval to the next, with no period of variation common to all intervals. The most prominent periods of the intervals range from 892 to 2283 days. The only prominent period for the entire data set was found to be 2016 days.

Acknowledgments. We thank hundreds of AAVSO observers worldwide who made about 27000 observations on RS Ophiuchi that made this study possible. B.D.O. gratefully thanks NASA Astrophysics Grant Supplements for Education, NAGW-3228 to the AAVSO, and the AAVSO Margaret Mayall Assistantship, which provided the funds for his high school summer internship in the AAVSO. We thank the National Science Foundation for Education Grant ESI-9154091, which supported the development by E.G. Foster, AAVSO technical staff, of the data analysis software used in this project.

\section{References}

Barbon, R., Mammano, A. \& Rosino, L. 1969, in Non-Periodic Phenomena in Variable Stars, L. Detre (Ed.), Academic Press (Budapest), p. 527

Campbell, L. 1920, Harvard Ann. 81, No. 5

Dobrzycka, D. \& Kenyon, S.J. 1994, AJ (in press)

Ferraz-Mello, S. 1981, AJ 86, 619

Garcia, M.R. 1986, AJ 91, 1400

Hack, M. \& Selvelli, P.L. 1993, in Cataclysmic Variables and Related Objects, M. Hack \& C. la Dous (Eds.), NASA SP-507 (Washington D.C.), p. 511.

Hjellming, R.M. et al. 1986, ApJ 305, L71

Pottasch, S.R. 1967, Bull. Astron. Inst. Netherlands 19, 227

Sanduleak, N. \& Stephenson, C.B. 1973, ApJ 185, 899

Tempesti, P. 1975, I.A.U. Inf. Bull. Variable Stars 974 


\section{Discussion}

K. Beuermann: Is the coverage sufficient to exclude an outburst between 1967 and 1985?

J. Mattei: Yes, there was no outburst.

F. Meyer: Accretion disks of the size of the disk in RS Ophiuchi should have regions of midplane temperatures around $10000 \mathrm{~K}$. One would expect therefore "dwarf nova outbursts", as found by Wolfgang Duschl (1986, A\&A $163,56)$ for symbiotic systems. 